\title{
Glass pipette-carbon fiber microelectrodes for evoked potential recordings
}

M.F.D. Moraes and

N. Garcia-Cairasco

\author{
Laboratório de Neurofisiologia e Neuroetologia Experimental, \\ Departamento de Fisiologia, Faculdade de Medicina de Ribeirão Preto, \\ Universidade de São Paulo, Ribeirão Preto, SP, Brasil
}

\author{
Correspondence \\ N. Garcia-Cairasco \\ Laboratório de Neurofisiologia e \\ Neuroetologia Experimental \\ Departamento de Fisiologia \\ FMRP, USP \\ 14049-900 Ribeirão Preto, SP \\ Brasil \\ Fax: 55 (016) 633-0017 \\ E-mail: ngcairas@fmrp.usp.br \\ Research supported by FAPESP \\ (No. 93/2023-2) and CNPq \\ (No. 521596/94).
}

Received October 8, 1996

Accepted September 25, 1997

\begin{abstract}
Current methods for recording field potentials with tungsten electrodes make it virtually impossible to use the same recording electrode also as a lesioning electrode, for example for histological confirmation of the recorded site, because the lesioning procedure usually wears off the tungsten tip. Therefore, the electrode would have to be replaced after each lesioning procedure, which is a very high cost solution to the problem. We present here a low cost, easy to make, high quality glass pipette-carbon fiber microelectrode that shows resistive, signal/noise and electrochemical coupling advantages over tungsten electrodes. Also, currently used carbon fiber microelectrodes often show problems with electrical continuity, especially regarding electrochemical applications using a carbon-powder/resin mixture, with consequent low performance, besides the inconvenience of handling such a mixture. We propose here a new method for manufacturing glass pipette-carbon fiber microelectrodes with several advantages when recording intracerebral field potentials.
\end{abstract}

The type of sensor used for electrophysiological recordings is of great importance. However, much effort is devoted to signal conditioning, while electrode performance is usually neglected $(1,2)$. The signal should naturally be amplified and filtered in order to minimize undesired noise, but an attempt should be made to avoid undesirable alterations of the recorded signal. This means that there should be a compromise between how much the signal can be "modified" and how "detailed" it needs to be for a refined experimental analysis. Improvement of biosensor performance reduces signal conditioning. Within this context, in the present report we describe an easy, high performance tech-
Key words

- Evoked potentials

- Glass pipette-carbon fiber

- Microelectrodes

- Voltammetry nique for the preparation of glass pipettecarbon fiber microelectrodes to be used for electrophysiological recordings (with considerable spatial resolution) and more specifically for auditory-evoked potentials (AEP) $(3,4)$.

Commonly used tungsten electrodes have the inconvenience of tip damage after being used for electrolytic lesioning, which is a very common practice in most of the experiments that require histological confirmation of the recorded site, because electrode tract is not always a good indicator of proper positioning. In the case of micropipettes their tracts are more difficult to visualize because the tips are usually in the micrometer range. 
Also, in experiments that use the recording electrode for stimulation, the electrode integrity is an important factor (e.g., extracellular impedance measurement) (5). The use of carbon fibers is interesting due to several intrinsic characteristics of this material such as low electrical resistance, high mechanical resistance (traction), low reactivity in an electrolytic solution, and high resistance to "heat", among others. In addition, as demonstrated here, they function properly after being used in electrolytic lesioning.

A technical difficulty when using carbon fiber microelectrodes is the establishment of electrical continuity between the fiber itself and the conductor to be fed into the amplifying/filtering system. The approach used in electrochemical experiments of voltammetry is the use of a carbon-powder/resin mixture, which seems to involve some technical difficulties as well as loss of quality in terms of electrical continuity (6). While serving the purpose of connecting the carbon fiber to an external conductor, the carbon-powder/resin mixture also serves a "mechanical" purpose by fixing the carbon fiber in place inside the glass micropipette. A special silver paint conductor is used as the electrical continuity medium in applications involving field potential recording $(2,7)$. The method presented here takes all of these basic requirements into account, yet with improved electrical characteristics. It is not the authors' idea to argue on the absolute best choice for glass pipette-carbon fiber microelectrode manufacturing, but rather to present a specific and reliable solution for electrophysiological recordings (e.g., sensory-evoked potentials).

The main difference in the methodology presented here is the use of $\mathrm{Hg}$ as electrical continuity material inside the micropipette. However, as mentioned above, a "mechanical" factor (fixing the carbon fiber in place inside the glass micropipette) was needed. Thus, we built a micropipette using a vertical microelectrode puller (Glass 2BBL, World Precision Instruments, Inc., New Haven, CT) and filled it with isopropylic alcohol to minimize electrostatic interaction between glass and carbon fiber by altering surface tension. We then inserted the desired number of carbon fibers to obtain an electrode tip diameter of about $15 \mu \mathrm{m}$. With a stereomicroscopic guide we heated the pipette tip by melting the glass over the carbon fiber and fixed it firmly to the micropipette (see Figure 1A). After these steps, the pipette itself was carefully heated in order to expel the isopropylic alcohol inside; if the carbon fiber was not properly "glued" to the glass, it would be expelled together with the liquid. The micropipette was then filled with $\mathrm{Hg}$ using a polyethylene tube and a syringe with care taken to avoid the formation of bubbles inside it, and a stainless steel wire was inserted (Figure 1B). The entire apparatus was then sealed with cyanoacrylate (Super Bond) at its open end, with the steel wire fixed in place.

In contrast to other studies on the same subject (6), all measurements were conducted considering the electrochemical coupling of the electrode. Briefly, a direct current (DC) of $0.1 \mathrm{~mA}$ was applied to the electrode while dipped in a pool of $0.9 \%$ saline, which behaved as the conducting medium. The voltage across the positive and negative terminals of the current source was measured and the resistance calculated by Ohm's law. Tone bursts of $40 \mathrm{~ms}(70 \mathrm{~dB}-3 \mathrm{kHz})$ repeated at a $2-\mathrm{Hz}$ frequency were applied unilaterally through hollowed stereotaxic auricular bars in anesthetized rats. The acoustic stimulation was synchronized with the start of the recording. Data were collected using a glass pipette-carbon fiber microelectrode $(15-\mu \mathrm{m}$ tip diameter, with $35 \mathrm{k} \Omega$ total resistance) for monopolar recording preamplified with a Princeton Applied Research 113 preamplifier (Princeton, NJ) (x 100 gain, $100 \mathrm{~Hz}-10$ $\mathrm{kHz}$ filter) and amplified with a CyberAmp 320 Axon Programmable Signal Conditioner (Foster City, CA) (x 100 gain, $100 \mathrm{~Hz}-6 \mathrm{kHz}$ filter). The reference electrode was a needle 


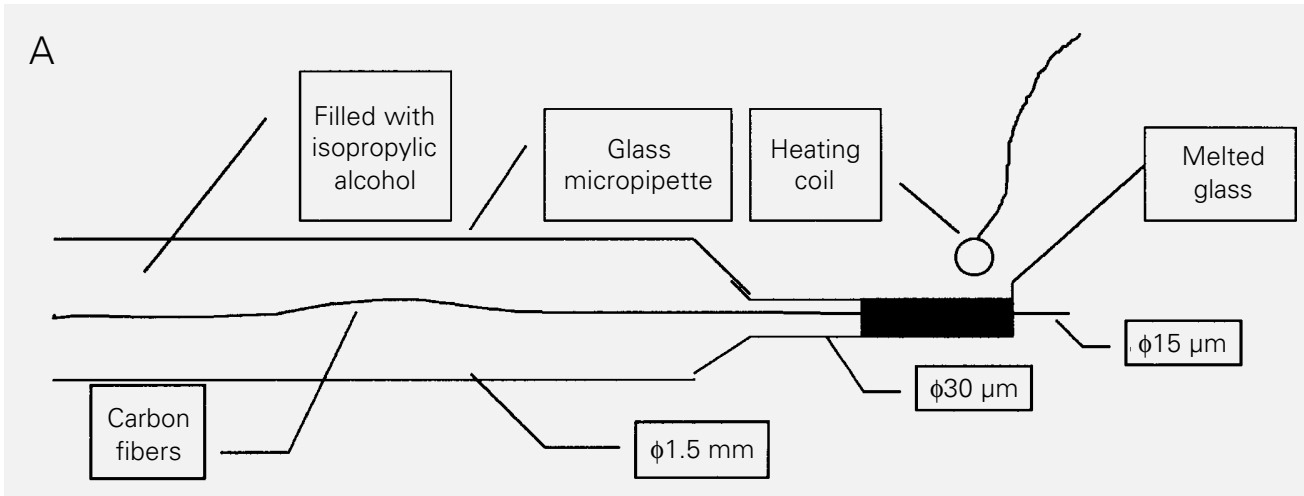

B

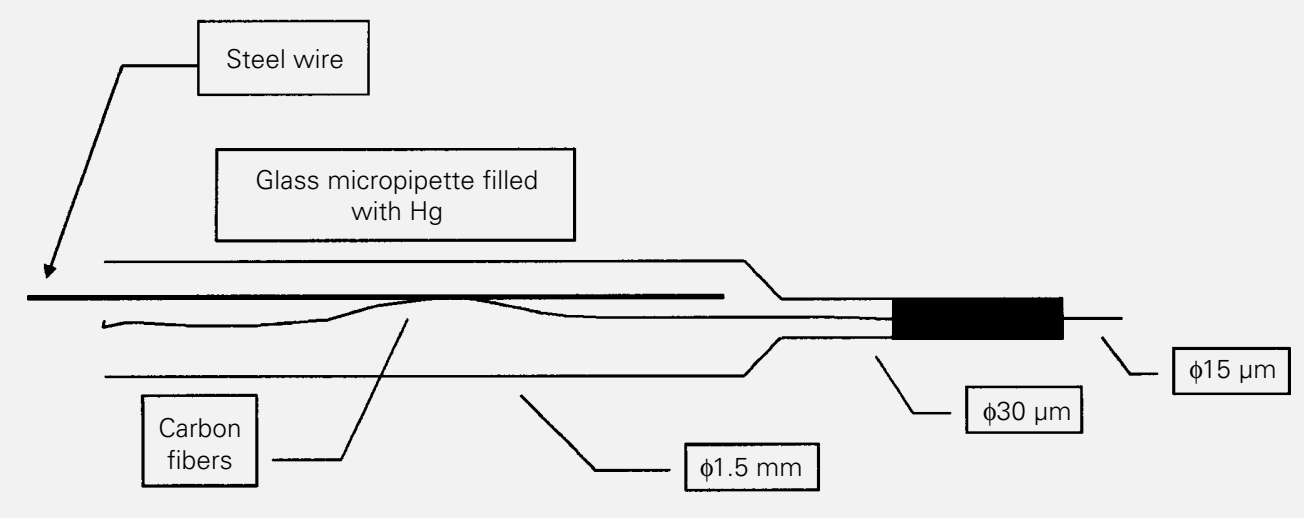

placed on the dorsal portion of the nasal bone. The first $20 \mathrm{~ms}$ of the AEP response were then converted by an A/D device (MP100, BIOPAC Systems, Inc., Goleta, $\mathrm{CA}$ ), with a $15-\mathrm{kHz}$ sampling rate, and averaged in groups of 50 recordings. A specific recording site in the central nucleus of the inferior colliculus of a Wistar rat was chosen for demonstration of electrode performance. Resistance was calculated by the method described previously, $\mathrm{R}=35 \mathrm{k} \Omega$. The AEP recording performance was maintained even after sequential lesions (data not shown). For comparison, as indicated by the manufacturer, the resistance of a tungsten electrode is $12 \mathrm{M} \Omega$ (measured in $1 \mathrm{kHz}$; A-M Systems, Everett, WA; 0.010"); therefore, there was a significant improvement of electrical resistance when compared to the current electrode (measured DC, $35 \mathrm{k} \Omega$ ). Since the electrode tip diameter observed was 15 $\mu \mathrm{m}$, we believe that there was no significant loss concerning spatial resolution of the recording site relative to an average neuron size of $50 \mu \mathrm{m}$. However, the "body" of the tungsten electrode is about 10 times larger than that of our carbon fiber microelectrode (tungsten: $250 \mu \mathrm{m}$, carbon fiber microelectrode: $30 \mu \mathrm{m}$ ), which is an important parameter when evaluating tissue damage caused by electrode placement. This is particularly important when doing extracellular recordings. Millar (8) pointed out that one of the misconceptions in extracellular recording is that high impedance electrodes are necessarily better for isolating cells than low-noise impedance ones. He comments that 1) this may indeed be true for intracellular recording, but not for extracellular studies. High impedance implies high noise, and one of the factors limiting the recording of extracellular spikes is the noise level of the electrode
Figure 1 - $A$, Schematic drawing of carbon fiber placement and fixation to a glass micropipette. All measurements were made using a digitizing table. $B$, Schematic drawing of wire placement and fixation. 
and amplifier system. 2) For intracellular electrodes, the impedance is related to tip diameter with high impedance implying a small diameter, and small tip electrodes will obviously do less damage to a cell when penetrating it than large ones. 3) For extracellular recordings, the tip diameter is often irrelevant, except for the fact that a large electrode does more general damage to the tissue during insertion.

In the present study, once the problem of electrical continuity was solved by means of the Hg filling, silver, nickel-chrome, stainless steel, copper, etc. could be used as the metal wire to be connected to the amplifying and recording devices. Stainless steel was used for cost purposes and for easy coupling with connectors. Isopropylic alcohol minimizes the electrostatic interaction of glass and carbon fibers because it alters surface tension and cools off immediately. Other substances are also used, such as acetone, for easier filling of glass pipettes with the carbon fibers. Vacuum suction is another alternative (9). Millar (8) always uses "wet" media to fill the pipettes such as acetone, ethanol or distilled water, because he noted that surface tension and forces due to static electricity make it practically impossible to insert the fibers "dry" into the blank (pipettes). Drying down excess fluid (8) and heating up the pipette tip, thus burning excess alcohol, as done in the present study, work similarly because in both cases the carbon fibers will firmly adhere to the inside of the pipette by surface tension. In our experiments we clearly noticed a good signal/noise ratio with only 50 averaged recordings, by observing baseline noise against AEP wave, even after the carbon fiber microelectrode was used for lesioning (data not shown). In the absence of proper equipment for stereomicroscopic aid when heating the microelectrode's tip (melting the "glass" around the carbon fiber), we observed that a well-trained "hand" can do fine by "quickly" passing the microelectrode's tip through a flame. One of the differences between the current electrode and those from other sources (8) is that in our experience the glass micropipette can be filled with carbon fibers before being built. The consequent problems are related to the sealing of the glass pipette tip to the carbon fiber tip and to the adjustment of the carbon fiber tip length with additional calculations of its caliber and resistance. Another important issue is how to connect the pipette with the amplification and recording devices. Millar (8) builds a glass micropipette with carbon fibers filling it with the heat of the puller being enough to insure a proper seal between the carbon fiber tip and the glass pipette. He cuts the protruding end of the fibers with small scissors and creates a dry contact with the internal part of the carbon fibers using a tin- or silver-coated copper wire and silver-doped conducting paint. When the wire makes contact with the carbon fibers, he places a drop of cyanoacrylate at the site of emergence of the insulation, sealing the connections.

The physical characteristics of our glass tubing-carbon fiber microelectrode permit precise electrode arrangements for simultaneous multi-site recordings. Figure 2A shows a three-channel AEP recording with anteroposterior intervals of $1 \mathrm{~mm}$ at a constant lateral position (Figure 2B) in a sagittal plane of the rat brain. Also, for experiments using chronically implanted electrodes, our laboratory uses silica tubing, which is commonly employed in microdialysis experiments (10), instead of glass pipettes since it presents a much better mechanical resistance with a considerable degree of flexibility. A typical AEP collected using such silica tubing electrodes is shown in Figure 2C. Other electrical characteristics of the electrode regarding electrochemical coupling should be investigated (11) in order to describe the nonlinearities of the "conductor"-electrolyte interface. However, as far as our current interests are concerned, the carbon fiber microelectrode presented here is well suited and 

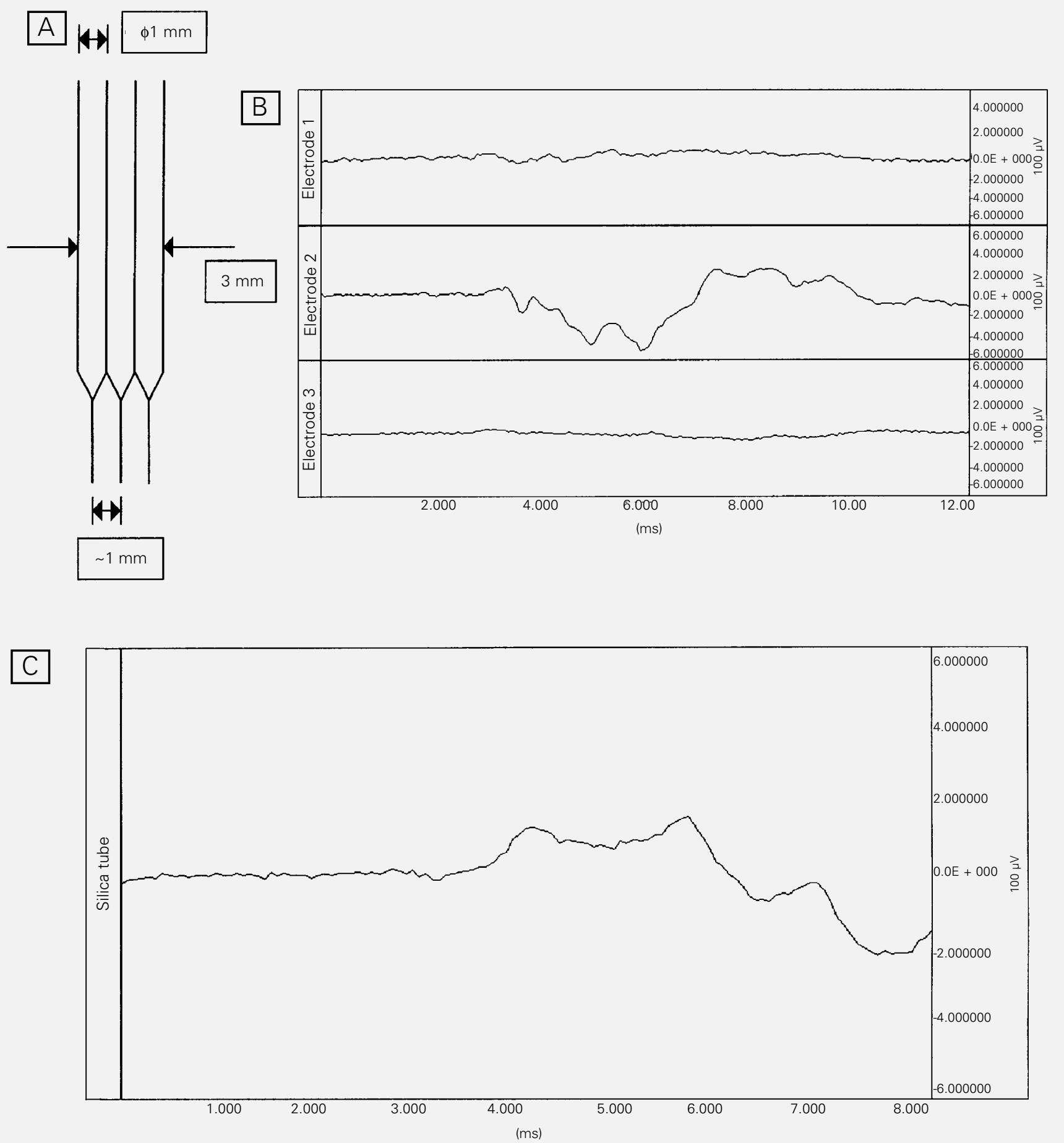

Figure 2 - A, Schematic diagram of a three-electrode array. B, Simultaneous three-channel recording with the electrode array of A. $C$, Auditory-evoked potential recording using a carbon fiber-silica tubing electrode. 
reliable for multiple AEP recordings with site labeling. Other applications of glass pipette-carbon fiber microelectrodes in addition to AEP recording are the complementary differential pulse voltammetry and fast cyclic voltammetry (9), as well as multi-unit activity recording, or a combination of voltammetry and unit activity recording (8), among others.

\section{References}

1. Stamford JA (1985). In vivo voltammetry: promise and perspective. Brain Research Reviews, 10: 119-135.

2. Millar J \& Williams GV (1988). Ultra-low noise silver-plated carbon-fibre microelectrodes. Journal of Neuroscience Methods, 25: 59-62.

3. Picton TW \& Hink RF (1974). Evoked potentials: How? What? and Why? American Journal of EEG Technology, 14: 9-44.

4. Scherg M, Vajsar J \& Picton T (1989). A source analysis of the human auditory evoked potentials. Journal of Cognitive Neuroscience, 1: 336-355.

5. Ranck Jr JB (1966). Electrical impedance. I. The subicular area of rats during paradoxical sleep. Experimental Neurology, 16: 416-437.
6. Math F \& Marianneau G (1994). A new method for manufacturing carbon-fibre microelectrodes. Journal of Neuroscience Methods, 52: 149-151.

7. Millar J (1992). Extracellular single and multiple unit recording with microelectrodes. In: Stamford JA (Editor), Monitoring Neuronal Activity - A Practical Approach. The Practical Approach Series. Oxford University Press, Oxford.

8. Millar J (1991). Simultaneous in vivo voltammetric and electrophysiological recording with carbon fiber microelectrodes. In: Conn M (Editor), Methods in Neuroscience. Vol. 4. Academic Press, New York, 143-154.
9. Stamford JA (1991). In vivo voltammetry. In: Conn M (Editor), Methods in Neuroscience. Vol. 4. Academic Press, New York, 127-142.

10. Doretto MC, Burger R, Mishra PK, GarciaCairasco N, Dailey JW \& Jobe PC (1994). A microdialysis study of amino acid concentration in the extracellular fluid of the substantia nigra of freely behaving GEPR9s: Relationships to seizure predisposition. Epilepsy Research, 17: 157-165.

11. Geddes LA, Da Costa CP \& Wise G (1970) The impedance of stainless steel electrodes. Medical and Biological Engineering, 9: 511-521 